\title{
Improvement of Subcutaneous Bioartificial Pancreas Vascularization and Function by Coencapsulation of Pig Islets and Mesenchymal Stem Cells in Primates
}

\author{
Sophie Vériter,* Pierre Gianello, * Yasuhiro Igarashi,* Gwen Beaurin,* Audrey Ghyselinck,* \\ Najima Aouassar, $\dagger$ Bénédicte Jordan, $\dagger$ Bernard Gallez, $\dagger$ and Denis Dufrane*ł \\ *Université Catholique de Louvain, Secteur des Sciences de la Santé, Institute of Clinical and Experimental Research, \\ Pôle de Chirurgie Expérimentale et Transplantation, Brussels, Belgium \\ $\dagger$ †niversity Clinical Hospital St Luc, Endocrine Cell Therapy Unit, Center of Tissular/Cellular Therapy, Brussels, Belgium \\ ¥Université Catholique de Louvain, Secteur des Sciences de la Santé, Louvain Drug Research Institute, \\ Biomedical Magnetic Resonance Research Group, Brussels, Belgium
}

\begin{abstract}
Insufficient oxygenation can limit the long-term survival of encapsulated islets in subcutaneous tissue. Transplantation of coencapsulated pig islets with adipose or bone marrow mesenchymal stem cells (AMSCs or BM-MSCs, respectively) was investigated with regard to implant vascularization, oxygenation, and diabetes correction in primates. The in vivo impact of MSCs on graft oxygenation and neovascularization was assessed in rats with streptozotocin (STZ)-induced diabetes that were subcutaneously transplanted with islets coencapsulated with AMSCs $(n=8)$ or BM-MSCs $(n=6)$. Results were compared to islets encapsulated alone $(n=8)$. STZ diabetic primates were subcutaneously transplanted with islets coencapsulated with BM-MSCs $(n=4)$ or AMSCs $(n=6)$. Recipients were monitored metabolically and immunologically, and neoangiogenesis was assessed on explanted grafts. Results were compared with primates transplanted with islets encapsulated alone $(n=5)$. The cotransplantation of islets with BM-MSCs or AMSCs in diabetic rats showed significantly higher graft oxygenation than islets alone $\left(3 \%\right.$ and $3.6 \% \mathrm{O}_{2}$ for islets + BM-MSCs or AMSCs, respectively, vs. $2.2 \%$ for islets alone). A significantly better glycated hemoglobin correction (28 weeks posttransplantation) was found for primates transplanted with islets and MSCs (7.4\% and 8.1\%, respectively) in comparison to islets encapsulated alone (10.9\%). Greater neoangiogenesis was found in the periphery of coencapsulated islets and AMSCs in comparison to islets alone $(p<0.01)$. In conclusion, the coencapsulation of pig islets with MSCs can improve significantly the islets' survival/function in vitro. The coencapsulation of islets with MSCs improves implant oxygenation and neoangiogenesis. However, the cotransplantation of islets with MSCs improves only slightly the long-term function of a subcutaneous bioartificial pancreas in a primate preclinical model.
\end{abstract}

Key words: Bioartificial pancreas; Mesenchymal stem cells (MSCs); Oxygenation; Islets

\section{INTRODUCTION}

Despite increasing efficacy rates $(1,17,23,28)$, islet transplantation remains limited by the need for chronic immunosuppression (tacrolimus, sirolimus, daclizumab), which is associated with serious side effects, including nephrotoxicity, hypertension, carcinogenicity, and hypersensitivity to infections $(14,22,27)$. Encapsulation of donor islets in a semipermeable matrix suppresses the need for lifelong immunosuppression. Immunoisolation protects encapsulated tissues against the recipient's immune system (both humoral and cellular immunities) while allowing passive diffusion of small molecules, including insulin, glucose, oxygen, and nutrients, among others.

A system of macroencapsulation in a monolayer, called the "Monolayer Cellular Device" (MCD) (9) (Dufrane, D., Gianello, P., Melvik, P. E. Alginate Coated, Collagen Matrix Cellular Device, Preparative Methods and Uses Thereof. Patent WO/2007/144389), demonstrated total correction of streptozotocin (STZ)-induced diabetes in five nonhuman primates for $\sim 6$ months in a model of "pig-toprimate" xenotransplantation (9). Two of these animals received second transplants, and diabetes was controlled for

Received April 26, 2012; final acceptance January 11, 2013. Online prepub date: February 4, 2013.

Address correspondence to Denis Dufrane, M.D., Ph.D., Université Catholique de Louvain, Institute of Experimental and Clinical Research, Pôle de Chirurgie Expérimentale et Transplantation, Avenue Hippocrate 55, bte B1.55.04, B-1200 Brussels, Belgium. Tel: +32 (0)2 7645587 ; Fax: +32 (0)2 76495 20; E-mail: denis.dufrane@uclouvain.be 
an additional 5 months (9). One explanation for this limited long-term diabetes correction could be the limited oxygen supply for encapsulated islets in subcutaneous tissue.

We previously demonstrated that the semipermeable membrane of the MCD, alginate PRONOVA SLM $1003 \%$ (sterile lyophilized high M alginate) (FMC BioPolymer AS $\mathrm{d} / \mathrm{b} / \mathrm{a}$ NovaMatrix, Drammen, Norway), is a biocompatible material allowing oxygenation levels that are adequate for the survival and function of islets implanted subcutaneously in a nondiabetic rat model $\left(\sim 5 \% \mathrm{O}_{2}\right)(30)$. Recently, we demonstrated that the diabetic state and oxygen consumption by encapsulated cells induce nonoptimal oxygen levels $\left(\sim 2 \% \mathrm{O}_{2}\right)$ for the survival and function of encapsulated islets (29). In addition, mesenchymal stem cells extracted from bone marrow (BM-MSCs) and adipose tissues (AMSCs) demonstrated proangiogenic properties by (i) in vitro secretion of vascular endothelial growth factor (VEGF) and (ii) in vivo, by improved subcutaneous MCD angiogenesis and, consequently, oxygenation during the early phase (1-4 weeks) posttransplantation $\left(\sim 4 \% \mathrm{O}_{2}\right)$ in an STZ-induced diabetic rat model (29).

Therefore, the aim of this study was to assess (i) in vitro: the impact of the BM-MSC or AMSC supplementation on islet survival, function, and oxygen consumption rate; (ii) in vivo: the impact of islet and BM-MSC or AMSC coencapsulation on implant oxygenation, vascularization, and diabetes control in STZ-induced diabetic rats and primates, for short- and long-term graft outcomes, respectively.

\section{MATERIALS AND METHODS}

\section{Animals}

Adult female Landrace pigs ( $>200 \mathrm{~kg}, n=8$ ) (Rattlerow Seghers, Lokeren, Belgium) were used as islet donors. AMSCs and BM-MSCs were harvested from female Landrace pigs weighing less than $60 \mathrm{~kg}$ ( $<6$ months old).

Coencapsulated islets and MSCs were transplanted into recipients with STZ-induced diabetes: (i) 14 male Wistar rats weighing 150 to $250 \mathrm{~g}$ (islets + BM-MSCs, $n=6$; islets +AMSCs, $n=8$ ) (local animalery) and (ii) 10 male cynomolgus monkeys ( $>3$ years old, $3-5 \mathrm{~kg}$ ) (islets + BM-MSCs, $n=4$; islets + AMSCs, $n=6$ ) (Hartelust, The Netherlands) (Fig. 1). The results obtained with these animals were compared to results previously published by our group on the transplantation of islets alone in Wistar rats (29) and primates (9).

The procedures were approved by the local Ethics Committee for Animal Care of the Université catholique de Louvain.

Pig BM-MSC and AMSC Isolation, Expansion, and Characterization

Pig BM-MSC and AMSC Isolation and Expansion. Pig BM-MSCs and AMSCs were isolated as previously described (29). Briefly, heparinized (LEO Pharmanv-sa, Lier, Belgium) bone marrow was mixed with a double volume of phosphate-buffered saline (PBS; Lonza, Verviers, Belgium) and layered over a Ficoll-Hypaque column (density: 1.077; Lymphoprep, Nycomed, Oslo, Norway). After centrifugation for $30 \mathrm{~min}$ at $1,250 \times \mathrm{g}$, the mononuclear cells were collected from the interface and washed in PBS at $450 \times g$ for $10 \mathrm{~min}$. The cells were placed in culture flasks (Gibco, Grand Island, NY, USA) in Dulbecco's modified Eagle's medium (DMEM) supplemented with $10 \%$ heat-inactivated fetal bovine serum (FBS) and antibiotics (penicillin $100 \mathrm{U} /$ $\mathrm{ml}$ and streptomycin $100 \mu \mathrm{g} / \mathrm{ml}$; all from Lonza) $(24,26)$.

Porcine fatty tissues were cut in a Petri dish and placed in collagenase (0.075 g; Sigma-Aldrich, St. Louis, MO, USA) reconstituted in Hank's balanced salt solution (with calcium and magnesium ions) at $37^{\circ} \mathrm{C}$ for $60 \mathrm{~min}$. After digestion, the collagenase was inactivated in DMEM supplemented with $10 \%$ heat-inactivated FBS, L-glutamine $(2 \mathrm{mM}$ ), and antibiotics (penicillin $100 \mathrm{U} / \mathrm{ml}$ and streptomycin $100 \mu \mathrm{g} / \mathrm{ml}$ ), all purchased from Lonza. Collected tissue was centrifuged for $10 \mathrm{~min}$ at $450 \times \mathrm{g}$. After filtration through a $500-\mu \mathrm{m}$ mesh screen (Haver, Battice, Belgium), the tissue was centrifuged for $10 \mathrm{~min}$ at $450 \times \mathrm{g}$ and then resuspended in proliferation medium made of DMEM supplemented with 10\% FBS and antibiotics (penicillin $100 \mathrm{U} / \mathrm{ml}$ and streptomycin $100 \mu \mathrm{g} / \mathrm{ml}$ ).

This initial passage of the primary cells was referred to as passage $0(\mathrm{P} 0)$. After 24 to $48 \mathrm{~h}$ of incubation at $37^{\circ} \mathrm{C}$ at $5 \% \mathrm{CO}_{2}$, cell cultures (both BM-MSCs and AMSCs) were washed with PBS and maintained in media up to P4 and then placed either in 24-well plates (Gibco) or on decellularized and lyophilized human fascia lata (i.e., human acellular collagen matrix) (7) for the assessment of the impact of MSC and islet coincubation on in vitro survival and function and in vivo improvement of oxygen tension and diabetes control.

In Vitro Characterization of BM-MSCs and AMSCs. BM-MSCs and AMSCs were stained with saturating amounts of monoclonal cluster of differentiation 90 (CD90), CD44, CD45, CD73, and CD105 antibodies (BD Pharmingen, San Diego, CA, USA) conjugated with phycoerythrin (PE) (CD44, CD45, CD90) or fluorescein isothiocyanate (FITC) (CD73, CD105). At least 10,000 events were analyzed by flow cytometry (FACScan, BD Biosciences, Erembodegem, Belgium) with CellquestPro software.

To confirm MSC multipotency, both bone marrow and adipose sources underwent adipogenesis, osteogenesis, and chondrogenesis by differentiation in specific adipocyte, osteoblast, and chondroblast induction media, respectively, as already described (25). Alzarin red (Sigma-Aldrich), Alcian blue (AppliChem GmbH, Darmstadt, Germany), and oil red (Sigma-Aldrich) staining were used to confirm osteogenic, chondrogenic, and adipogenic phenotypes after differentiation. 


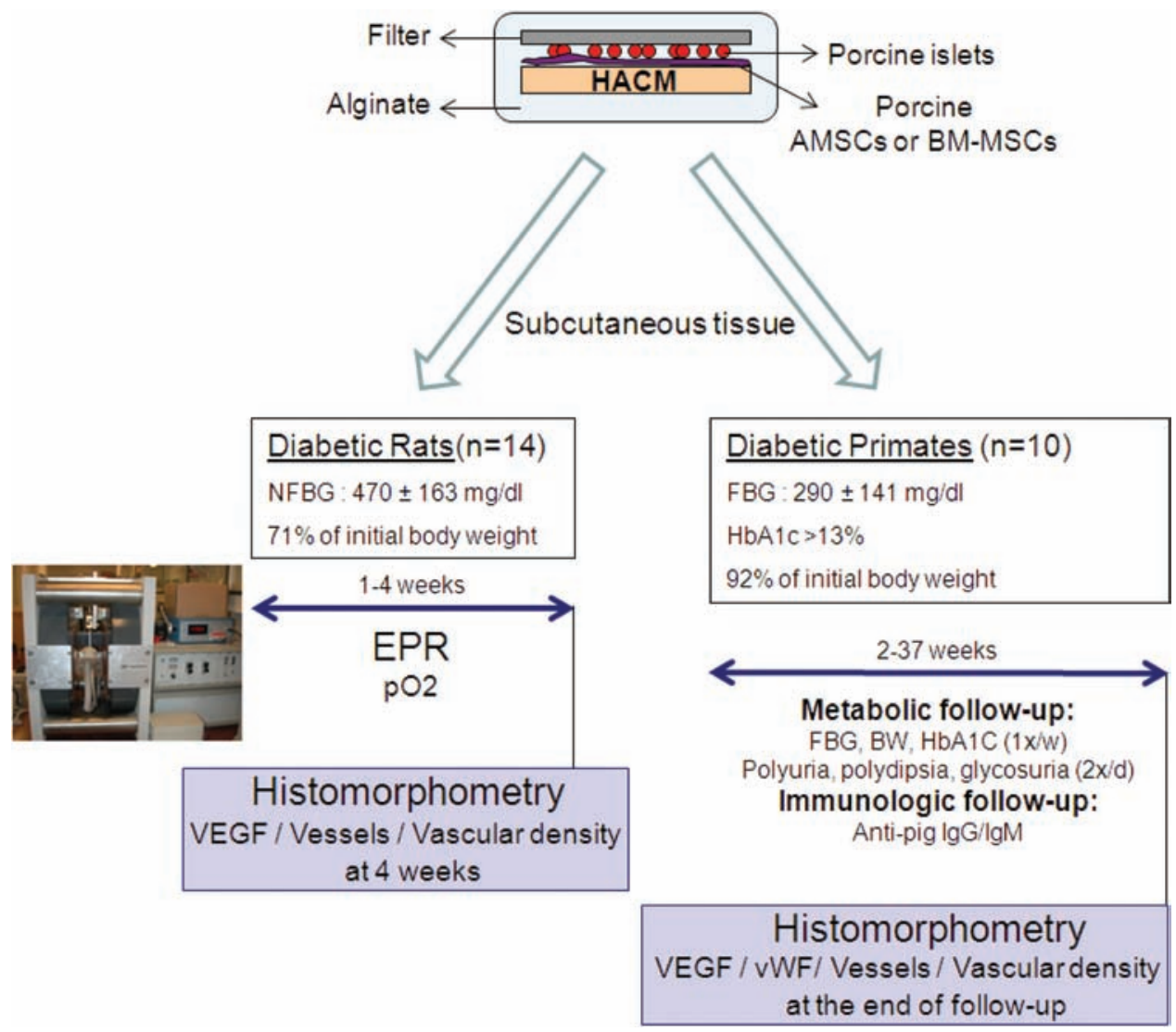

Figure 1. Protocol for in vivo assessment of the impact of MSCs on monolayer cellular device (MCD) oxygenation, neovascularization, survival, and function. Porcine BM-MSCs and AMSCs were coencapsulated with pig islets in MCDs and implanted subcutaneously in (i) 14 diabetic rats and (ii) 10 diabetic nonhuman primates to improve angiogenesis and islet survival. Oxygenation of MCDs implanted in rats was followed weekly for 4 weeks by EPR. Metabolic and immunologic courses were assessed up to 37 weeks posttransplantation. Neovascularization in the MCD periphery was assessed after graft explantation (4 weeks posttransplantation for rats, up to 37 weeks posttransplantation for primates). HACM, human acellular collagen matrix; BM-MSCs, bone-marrow mesenchymal stem cells; AMSCs, adipose mesenchymal stem cells; NFBG, nonfasting blood glucose; FBG, fasting blood glucose; HbA1c, glycated hemoglobin, EPR, electronic paramagnetic resonance; BW, body weight; VEGF, vascular endothelial growth factor; vWF, von Willebrand's factor.

Pig Islet Isolation. Pig islets were isolated following a previously described method (8). Adult pigs were euthanized at a local slaughterhouse (Centre A. de Marbaix, Louvain-la-Neuve, Belgium). Cerebral death was obtained by penetrating bolt gun. Pigs were exsanguinated, and the pancreases were harvested, placed on ice, and perfused with cold modified University of Wisconsin (UW) solution (no hydroxyethyl starch and low $\mathrm{K}^{+} / \mathrm{high} \mathrm{Na}^{+}$; allopurinol, D-(+)-raffinose pentahydrate, glutathionefree acid reduced, lactobionic acid-free acid, potassium phosphate monobasic, magnesium sulfate; all from Sigma-Aldrich) (8). The warm ischemia time varied from 11 to $40 \mathrm{~min}$. The pancreases were placed in a box with modified UW solution at $4^{\circ} \mathrm{C}$ for the transport to the lab. The cold ischemia time varied from 60 to $171 \mathrm{~min}$.

After being completely dissected, each pancreas was subjected to modified static digestion. After being infused with Liberase DL Research Grade (Roche/Boehringer Mannheim, Brussels, Belgium; $0.43 \mathrm{mg} / \mathrm{ml}$ ) dissolved in modified UW solution, the pancreas was placed in a sterile 1-L Nalgene jar (Nalgene, Rochester, NY, USA) for digestion by static incubation at $37^{\circ} \mathrm{C}$ for $30-45 \mathrm{~min}$. After filtration through a $500-\mu \mathrm{m}$ filter and purification in a Ficoll gradient (densities 1.11, 1.096, 1.06; Mediatech, Manassas, VA, USA), the islets were suspended in HamF10 (Gibco) + 10\% newborn calf serum (Merck-Eurolab, Overijse, Belgium).

\section{In Vitro Effect of MSC Incubation With Islets}

Effect of MSCs on Islet Oxygen Consumption. The impact of MSCs on total cellular oxygen consumption was assessed in vitro by electronic paramagnetic resonance (EPR) on a Bruker EMX EPR spectrometer (Ettlingen, Germany) operating at $9 \mathrm{GHz}(5,15)$. 
Oxygen consumption by pig islets alone, AMSCs and BM-MSCs alone, or islets coincubated with AMSCs or BM-MSCs at both $5 \mathrm{mM}$ (normoglycemic) and $20 \mathrm{mM}$ (hyperglycemic) glucose (Baxter, Lessines, Belgium) was evaluated. One hundred microliters of culture medium [Rose Park Memorial Institute (RPMI) medium (Lonza) containing 10\% heat-inactivated fetal calf serum, $100 \mathrm{IU} / \mathrm{ml}$ penicillin, $100 \mu \mathrm{g} / \mathrm{ml}$ streptomycin, 5 or $20 \mathrm{mM}$ glucose (G5 and G20, respectively)] containing islets (20,000 islets), MSCs (500,000 cells), or islets + MSCs $(20,000$ islets $+500,000$ MSCs $)$ were suspended in $10 \%$ dextran [dextran from Leuconostoc mesenteroides (molecular weight: 65,000-76,000), Sigma-Aldrich] in a complete medium in the presence of neutral nitroxide, $15 \mathrm{~N}$ 4-oxo-2,2,6,6-tetramethylpiperidine-d16-15N-1-oxyl at $0.2 \mathrm{mM}$ (CDN Isotopes, Pointe-Claire, Quebec, Canada) as an oxygen probe. Glass capillaries were filled with this preparation. The probe was previously calibrated so that the line width measurements could be related to $\mathrm{O}_{2}$ concentration. The oxygen consumption by total incubated cells was thereby evaluated.

The sealed tubes were placed into quartz EPR tubes (hematocrit tubes, Hirschmann, Eberstadt, Germany), and the samples were maintained at $37^{\circ} \mathrm{C}$. Oxygen pressure $\left(\mathrm{pO}_{2}\right)$ measurements were performed each minute over a 10 -min period. With the resulting line width reports on $\mathrm{pO}_{2}$, it was possible to calculate oxygen consumption rates by measuring the $\mathrm{pO}_{2}$ in the closed tube as a function of time and to subsequently compute the slope ( $k$ value) of the resulting plot. The area under the curve (AUC) was also calculated as the sum of the surface area between each point.

Three independent tests per situation were performed.

Effect of MSCs on Islet Survival and Function. To assess the impact of MSCs on islet survival and function, BM-MSCs and AMSCs were cocultured with freshly isolated islets. The viability and function were assessed after $48 \mathrm{~h}$.

Islet Viability. One thousand isolated pig islets $(24 \mathrm{~h}$ after isolation) were placed in 24-well plates and cultured alone or with confluent pig BM-MSCs or AMSCs. Cells were cultured for $48 \mathrm{~h}$ in $1 \mathrm{ml}$ of RPMI medium containing $10 \%$ heat-inactivated FBS, $100 \mathrm{IU} / \mathrm{ml}$ penicillin, and $100 \mu \mathrm{g} / \mathrm{ml}$ streptomycin with glucose concentrations of $5 \mathrm{mM}$ (normoglycemic) or $20 \mathrm{mM}$ (hyperglycemic).

Islet viability was assessed by dithizone (DTZ) staining in function of islet size evaluated by morphometrical analysis.

After $48 \mathrm{~h}$ of incubation, supernatant was removed and replaced by DTZ solution (Merck, Darmstadt, Germany). The numbers of islets were counted in five nonoverlapping areas per well (magnification 10×). After capture of pictures of wells, ImageJ 1.43i software (NIH, Bethesda,
MD, USA) was used to quantify the proportion of the area (in pixels ${ }^{2}$ ) occupied by islets in three nonoverlapping areas per well. Mortality of islets was assessed after trypan blue (Lonza, Walkersville, MD, USA) staining. Islets were examined microscopically in three nonoverlapping areas (magnification $10 \times$ ) and classified into five categories $(<25 \%, 26-49 \%, 50-74 \%, 75-99 \%, 100 \%$ of viable cells per islet). The proportion of islets in each category was thereafter quantified, and islets were classified as "bad islets" (BI) ( $<25 \%$ viable cells per islet) and "good islets" (GI) (>75\% viable cells per islet).

Experiments were performed in triplicate.

Islet Function. The impact of islet and MSC coincubation on islet function was assessed in vitro. Porcine BM-MSCs and AMSCs were cultured in 12-well culture plates (Gibco) until confluence was reached. After isolation and overnight culture in Ham-F10 medium, 100 handpicked porcine islets were incubated alone or with BM-MSCs or AMSCs for $48 \mathrm{~h}$ in $2 \mathrm{ml} \mathrm{RPMI}$ at $5 \mathrm{mM}$ glucose. Function of islets was thereafter assessed by 24-h incubation in $2 \mathrm{ml} \mathrm{RPMI}$ at 5 (G5), $15 \mathrm{mM}$ glucose (G15), and $15 \mathrm{mM}$ glucose $+1 \mu \mathrm{M}$ forskolin (Fsk) (G15+Fsk) (Calbiochem-Behring, San Diego, CA, USA) (added from a millimolar stock solution in dimethyl sulfoxide; Sigma-Aldrich). Six replicates per time and tested glucose concentration were performed. Media were thereafter recovered for insulin quantification, and islets were transferred in acid-ethanol for extraction and quantification of hormones by radioimmunoassay (Human-specific RIA kit; Millipore, Billerica, MA, USA). A cross-reaction of $100 \%$ between human and porcine insulin was described by the manufacturer for RIA kits.

\section{In Vivo Effect of MSC Cotransplantation With Islets}

The effects of islet and MSC coencapsulation on implant oxygenation and graft function and survival were assessed in vivo in diabetic rats and primates, respectively (Fig. 1).

STZ-induced $(55 \mathrm{mg} / \mathrm{kg})$ diabetic Wistar rats weighing 150 to $250 \mathrm{~g}$ (nonfasting blood glucose greater than $240 \mathrm{mg} / \mathrm{dl}$ for 4 consecutive weeks) were subcutaneously transplanted with pig islets coencapsulated with AMSCs $(n=8)$ or BM-MSCs $(n=6)$. Oxygen pressures inside implants were followed weekly for 4 weeks. Results were compared with diabetic rats previously grafted with islets encapsulated alone $(n=8)(29)$.

STZ-induced diabetic primates (single to multiple doses of $50 \mathrm{mg} / \mathrm{kg}$ ) were transplanted with pig islets coencapsulated with pig BM-MSCs $(n=4)$ or pig AMSCs $(n=6)$. Metabolic and immunological courses were monitored to the point of implant dysfunction. In addition, diabetic state was monitored after graft removal. Neoangiogenesis of 
explanted grafts was finally assessed. Results were compared with those from primates previously transplanted with islets encapsulated alone $(n=5)(9)$.

Primates [mean fasting blood glucose (FBG) at $66 \mathrm{mg} /$ $\mathrm{dl}$ and mean glycated hemoglobin (HbAlc) at $5.7 \%$, mean body weight at $4.2 \mathrm{~kg}$ ] were rendered diabetic by single or repeated intravenous injections of $50 \mathrm{mg} / \mathrm{kg} \mathrm{STZ}$ as previously described (12). A FBG $>150 \mathrm{mg} / \mathrm{dl}$ and $\mathrm{HbAlc}>13 \%$ associated with glycosuria, polyuria, and polydipsia demonstrated the diabetic state. In addition, anti-insulin immunohistochemistry was performed on primate pancreases at the end of the experiment to confirm the destruction of endogenous $\beta$-cells.

Coencapsulation and Transplantation of Pig Islets and MSCs. MCDs composed of islets and MSCs were prepared as described previously $(9,29)$. Both BM-MSCs and AMSCs cultivated up to confluence on human acellular matrix (HACM) were coencapsulated with 125 to 300 freshly isolated pig IEQs/g (for diabetic rats) and 15,000 to 62,500 freshly isolated pig IEQs/kg (for diabetic primates) with 3\% w/v SLM-100 alginate (Batch 110064, FMC BioPolymer, Drammen, Norway) containing a high concentration of mannuronic acid (high-M; 56\%) and a low endotoxin level (endotoxin $<25 \mathrm{EU} / \mathrm{g}$ ). In a previously published study primates were transplanted with 16,000 to 33,500 IEQs/kg encapsulated alone (9).

Diabetic rats were transplanted with coencapsulated pig islets and BM-MSCs or AMSCs in small subcutaneous pockets located in a right paravertebral site. Diabetic primates were transplanted with pig islets coencapsulated with BM-MSCs or AMSCs in abdominal and dorsal subcutaneous tissues.

In Vivo Graft Oxygenation in Diabetic Rats. The $\mathrm{pO}_{2}$ inside the MCD was studied for up to 4 weeks after transplantation under gas anesthesia (isoflurane; FORENE ${ }^{\circledR}$, Wavre, Belgium), which preserves tissue oxygenation (2). An EPR spectrometer (Magnettech, Berlin, Germany) equipped with a low-frequency microwave bridge operating at $1.2 \mathrm{GHz}$ and an extended loop resonator (1-cm depth sensitivity) was used for $\mathrm{pO}_{2}$ measurements (13).

At 1,2,3, and 4 weeks after implantation, $\mathrm{pO}_{2}$ inside the grafts was monitored. Rats were anesthetized and placed in the EPR spectrometer, with the region of graft implantation positioned under the surface coil. EPR spectra were recorded with a modulation amplitude less than one third of the peak-to-peak line width.

\section{In Vivo Graft Assessment in Diabetic Primates}

Metabolic Course After Transplantation. Levels of FBG (Accu-Chek sensor, Mannheim, Germany), HbA1c concentrations [MetricaA1c Now+ diagnostic kit (Euromedix NV, Leuven, Belgium)], and body weight were followed weekly. Polyuria, polydipsia (measure of the volume), and glycosuria [VetstiX(R): Melet Schloesing Laboratoires, Osny, France] were monitored twice a day.

In addition, the graft function was assessed by performing an intravenous glucose tolerance test (IVGTT) prior to diabetes induction, before transplantation, and at a mean of 28 weeks postimplantation (islets + MSCs) or 22 weeks postimplantation (islets alone) (9). A dose of $0.5 \mathrm{~g} / \mathrm{kg}$ body weight $50 \% \mathrm{w} / \mathrm{v}$ glucose was injected intravenously. Blood samples were taken before and 1, 6 , 10, 20, 30, and 60 min after glucose injection for serum glucose and insulin quantification by radioimmunoassay (insulin-specific RIA kit, Millipore).

Humoral Response After Transplantation. Detection of anti-porcine $\operatorname{IgM}$ and $\operatorname{IgG}(9,10)$ : Specific anti-porcine $\operatorname{IgM}$ and $\mathrm{IgG}$ antibodies were detected in primate sera by flow cytometry using a fluorescence-activated cell sorter (pretransplantation and 14, 30,60, and 90 days posttransplantation for IgM; pretransplantation and 30, 60, 90, and 120 days posttransplantation for $\mathrm{IgG}$ ). Two hundred thousand pig PBMCs were used as specific targets and incubated for $20 \mathrm{~min}$ at $4^{\circ} \mathrm{C}$ with $100 \mu \mathrm{l}$ of primate serum (from our own primates; diluted 1/20). After washing, $100 \mu \mathrm{l}$ of anti-primate $\operatorname{IgM}$ (Lo-BM2) or IgG (Lo-hG-22) fluorescein isothiocyanate-conjugated antibodies (diluted 1/100; Université catholique de Louvain, Experimental Immunology Unit, Brussels, Belgium) was added and incubated for $20 \mathrm{~min}$ at $4^{\circ} \mathrm{C}$. Cells were then washed and counted (FACScan) with CellquestPro software.

$\alpha$-Galactosyl ( $\alpha$-Gal)-enzyme-linked immunosorbent assay: The anti- $\alpha-G a l$ specificity was characterized by enzyme-linked immunosorbent assay (ELISA) pretransplantation and at 7, 30, 60, 90, and 120 days posttransplantation.

Flat-bottomed, 96-well Maxisorb plates (NUNC A/S, Roskilde, Denmark) were coated overnight at $4^{\circ} \mathrm{C}$ with $0.05 \mu \mathrm{g}$ of human serum albumin $\alpha$-galactosyl (Dextra Laboratories Ltd., Reading, UK). Fifty microliters of primate serum (diluted 1/10) was then added and incubated for $1 \mathrm{~h}$ at $37^{\circ} \mathrm{C}$. After washing in PBS-Tween (Merck, Darmstadt, Germany), the plates were incubated for $1 \mathrm{~h}$ at $37^{\circ} \mathrm{C}$ with $100 \mu \mathrm{l}$ of anti-primate $\mathrm{IgG}$ (Lo-hG-22) peroxidase-conjugated antibodies (diluted 1/100; Université catholique de Louvain, Experimental Immunology Unit). The plates were then washed and developed for 3-5 min with ortho-phenylenediamine (Sigma-Aldrich, Bornem, Belgium), and absorbance at $492 \mathrm{~nm}$ was measured (9).

Cytotoxicity of anti-pig IgG antibodies: The cytotoxicity level of anti-pig antibodies found in primate sera was determined by flow cytometry complement-mediated cytotoxicity assay at 30 and 120 days posttransplantation. Five hundred thousand pig PBMCs were used as specific targets and incubated for $30 \mathrm{~min}$ at $4^{\circ} \mathrm{C}$ with $100 \mu \mathrm{l}$ of heat-inactivated primate serum. After two washings in 
PBS, $0.05 \%$ azide (w/v) (Merck, Darmstadt, Germany), $1 \%$ bovine serum albumin (BSA, v/v; Sigma-Aldrich, Bornem, Belgium), cells were incubated for $30 \mathrm{~min}$ at $37^{\circ} \mathrm{C}$ with rabbit complement (AbD Serotec, Düsseldorf, Germany). After two washings in PBS-azide $0.05 \%-B S A$ $1 \%, 1.6 \mu \mathrm{g}$ of propidium iodide (Sigma-Aldrich) was added and incubated in the dark for $15 \mathrm{~min}$ at room temperature. Cells were counted (FACScan) with Cellquest Pro software after one step of washing. The percentage of cytotoxicity was calculated as $100 \times[1-(\%$ living cells after incubation with heat inactivated "test" serum-rabbit complement $) /(\%$ living cells after incubation with the corresponding heat-inactivated "pretransplant" serum- rabbit complement)] (20).

Assessment of Implant/Pancreas Hormonal Content and Implant Neovascularization. After graft removal, explanted grafts as well as rat and primate pancreases underwent histological examination. After overnight fixation in formol (VWR International SA, Leuven, Belgium) at room temperature, explants and pancreases were embedded in paraffin and cut into 5- $\mu$ m-thick sections. Sections of MCDs were thereafter routinely colored with hemalun-eosin (Merck) to assess the presence of MSCs in the explanted MCDs. Sections of MCDs and pancreas were immunohistologically stained for insulin (1:1,200; Abcam, Cambridge, UK) and glucagon (1:100; BD Biosciences) to characterize the hormonal content.

Tissues surrounding the grafts were also taken to assess the neovascularization of implants. Masson's trichrome staining (Merck) was performed to determine the degree of angiogenesis and vascular density by image analysis with Image J 1.43i software, which was used to quantify the proportion of the area (in pixels ${ }^{2}$ ) occupied by vessels in three nonoverlapping counting areas per slide.

Immunostainings of VEGF (1:50; Clone A20, Santa Cruz Biotechnology, Inc., Santa Cruz, CA, USA) and von Willebrand factor (vWF) (factor VIII) (for primate tissues only) (1:1,000; DAKO, Enschede, The Netherlands) were performed by immunohistochemistry.
The number of vessels, as well as the number of VEGFand factor VIII-positive cells per area was quantified by histomorphometry in 10 nonoverlapping areas per slide (original magnification $25 \times$ with a $400 \times 400-\mu \mathrm{m}^{2}$ grid).

\section{Statistics}

Values are presented as means \pm SD except when otherwise specified. The one-sample Kolmogorov-Smirnov test was used to assess the normal distribution of values. The statistical significance of differences between more than two experimental groups with a normal distribution was tested by one-way analysis of variance with a Bonferroni post hoc test; groups with nonnormal distribution were analyzed by Kruskal-Wallis tests. The statistical tests were carried out with Systat version 8.0. Differences were considered to be significant at $p<0.05$.

\section{RESULTS}

\section{In Vitro Pig BM-MSC and AMSC Characterization}

BM-MSCs and AMSCs were confirmed as stem cells by CD90, CD44, CD45, CD73, and CD105 expression by flow cytometry and differentiation in adipocytes, osteocytes, and chondrocytes as recommended (6).

Both undifferentiated BM-MSCs and AMSCs presented a fibroblast phenotype in the proliferation medium. In contrast, the osteoinduction was confirmed by alzarin red staining as well as chondrogenic and adipogenic phenotype (after differentiation) by alcian blue and oil red stainings, respectively.

\section{In Vitro Assessments}

Effect of MSCs on Islets Oxygen Consumption. Oxygen consumption of AMSCs, BM-MSCs, and islets (alone or in coincubation) was investigated in vitro to assess the impact of MSC coencapsulation on islet oxygen consumption in normoglycemia ( $5 \mathrm{mM}$ glucose) and hyperglycemia (20 mM glucose). MSC source or the glycemic state did not significantly influence the rate of oxygen consumption during $10 \mathrm{~min}$ ( $k$ values of -4.6 and -7.9 ,

\section{FACING PAGE}

Figure 2. Assessment of oxygen consumption rate and impact of MCSs and islets coculture on islet survival and function. (A) Oxygen consumption rate of BM-MSCs and AMSCs at $5 \mathrm{mM}$ glucose. A significantly higher oxygen consumption rate (AUC, area under the curve) was found for AMSCs alone, AMSCs + islets, and BM-MSCs + islets in comparison to islets alone and BM-MSCs alone $(* p<0.05)$. (B) Oxygen consumption rate of BM-MSCs and AMSCs at $20 \mathrm{mM}$ glucose. A significantly higher AUC was calculated for AMSCs alone or islets coincubated with AMSCs in comparison with islets alone and islets coincubated with BM-MSCs (\#p<0.05). A significantly higher AUC was found for islets coincubated with BM-MSCs in comparison with islets alone $(\$ p<0.05)$. (C) Impact of MSCs and islet coincubation on islet survival after $48 \mathrm{~h}$ of incubation with $5 \mathrm{mM}$ glucose. Coincubation of islets with AMSCs showed a lower proportion of bad islets (BI) and a higher proportion of good islets (GI) in comparison to islets alone $\left({ }^{*} p<0.05\right)$. (D) Impact of MSCs and islet coincubation on islet survival after $48 \mathrm{~h}$ of incubation with $20 \mathrm{mM}$ glucose. A higher proportion of BI was found with islets alone in contrast with the coincubation of islets with AMSCs $\left({ }^{\circ} p<0.05\right)$. A higher proportion of GI was found with islets coincubated with AMSCs alone in comparison to islets alone or islets coincubated with BM-MSCs $(\$ p<0.05)$. (E) Impact of MSCs and islet coincubation on insulin stimulation index. Higher stimulation indexes were found for islets coincubated with AMSCs in comparison to islets alone and islets coincubated with BM-MSCs. (F) Impact of MSCs and islet coincubation on islet insulin content. Stable insulin content was found in islets coincubated with AMSCs in contrast with decreased insulin content upon glucose stimulation for islets alone and islets coincubated with BM-MSCs. G15 +Fsk $=15 \mathrm{mM}$ glucose $+1 \mu \mathrm{M}$ forskolin; G5 =5 mM glucose. 
-2.6 and -2.3 , and -6.4 and -3.6 for AMSCs, BM-MSCs, and islets alone and in $5 \mathrm{mM}$ and $20 \mathrm{mM}$ glucose, respectively). The addition of MSCs to islets did not significantly increase the rate of oxygen consumption ( $k$ values of -9.9 and $-9.1 ;-8.3$ and -6.7 for islets +AMSCs or islets + BM-MSCs at $5 \mathrm{mM}$ and $20 \mathrm{mM}$ glucose, respectively). However, AMSCs alone, AMSCs + islets, and BM-MSCs + islets showed a significantly higher area under the curve (AUC) in comparison to islets alone and BM-MSCs alone (718.9, 1042.5, and 841.2 vs. 102.8 and 300.4 normalized $\% \mathrm{O}_{2} / \mathrm{min}$, respectively) at $5 \mathrm{mM}$ glucose (Fig. 2A), indicating a higher $\mathrm{O}_{2}$ consumption. In hyperglycemic conditions, a higher AUC was found for AMSCs alone and AMSCs + islets in comparison to islets alone and BM-MSCs (841.8 and 869.0 vs. 245.9 and 460.3 normalized $\% \mathrm{O}_{2} / \mathrm{min}$, respectively) (Fig. 2B).
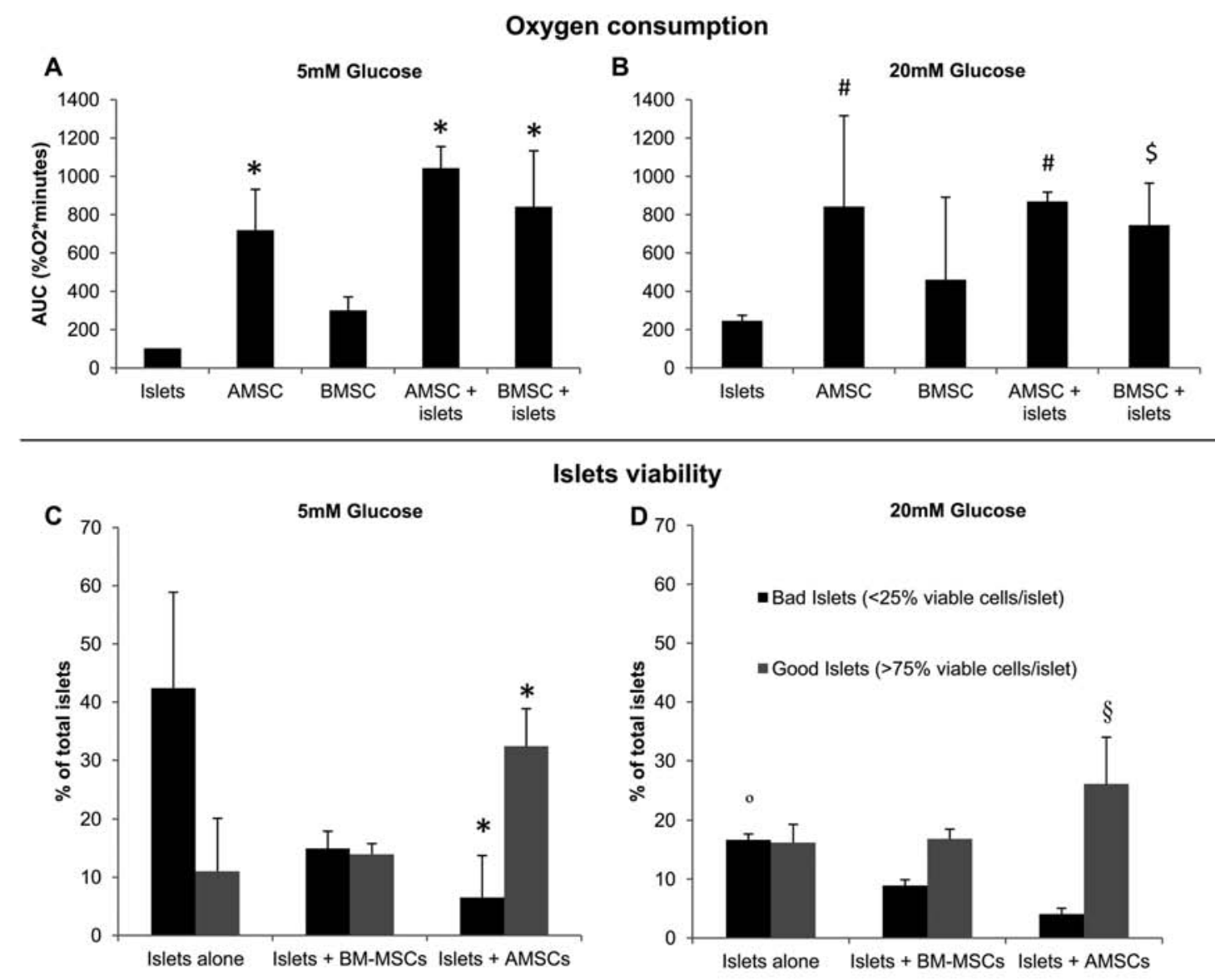

slets viability

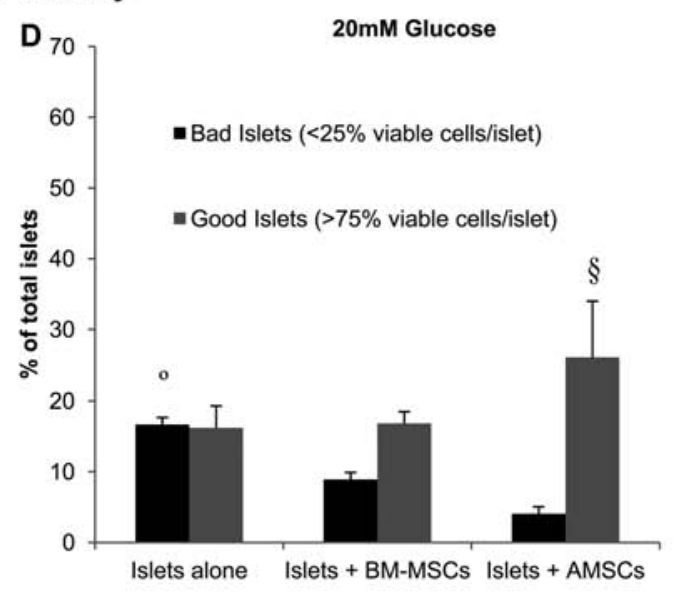

Islets functionality

E

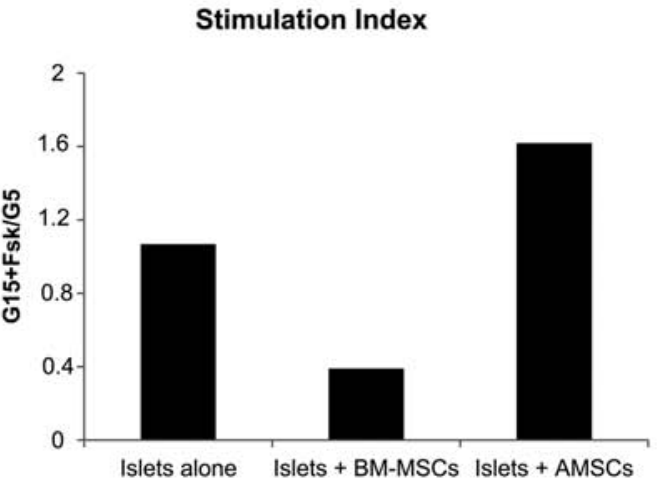

$\mathbf{F}$

Insulin Content at G15+Fsk

(\% vs G5)

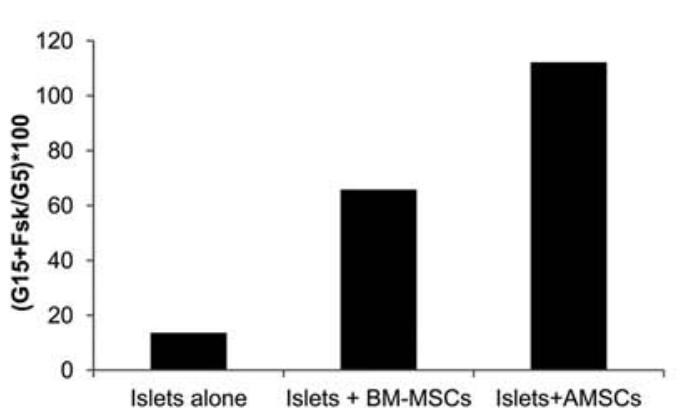


BM-MSCs + islets also showed a significantly higher AUC in comparison to islets alone (841.1 vs. 245.9 normalized $\% \mathrm{O}_{2} /$ min, respectively).

\section{Effect of MSCs on Islets Survival and Function}

Islets Survival. Islets were classified as BI $(<25 \%$ viable cells per islet) or GI ( $>75 \%$ viable cells per islet). After $48 \mathrm{~h}$ of incubation with $5 \mathrm{mM}$ glucose, better cellular survival was found for islets coincubated with AMSCs than islets alone as shown by a lower proportion of BI and a higher proportion of GI in comparison to islets alone $(p<0.05)$ (Fig. 2C).

Islets coincubated with AMSCs in hyperglycemic conditions also showed a lower proportion of $\mathrm{BI}$ and a higher proportion of GI than islets alone $(p<0.05)$ (Fig. 2D).

After $72 \mathrm{~h}$ of incubation with $5 \mathrm{mM}$ glucose, islets alone showed a higher proportion of $\mathrm{BI}$ in comparison to islets coincubated with AMSCs/BM-MSCs $(p<0.05)$ (data not shown).

A higher proportion of $\mathrm{BI}$ was found for islets alone in comparison to islets + AMSCs or BM-MSCs at $20 \mathrm{mM}$ glucose after $72 \mathrm{~h}$ of incubation $(p<0.005)$. In contrast, a higher proportion of GI was found for islets + AMSCs in comparison to islets coincubated with BM-MSCs and islets alone $(p<0.01)$ (data not shown).

A significant decrease in islet number was gradually found (for each experimental group) between 24 and $72 \mathrm{~h}$ of culture $(p<0.05)$.

Significantly larger islets were found at $48 \mathrm{~h}$ in comparison to 24 and $72 \mathrm{~h}$ for islets coincubated with BM-MSCs or AMSCs at $5 \mathrm{mM}$ glucose in comparison to islets alone $(p<0.01)$. In contrast, significantly larger islets were found at $24 \mathrm{~h}$ of culture with BM-MSCs at $20 \mathrm{mM}$ glucose versus 48 and $72 \mathrm{~h}(p<0.05)$.

Islets Function (Insulin Production). After glucose stimulation during $24 \mathrm{~h}$, significantly higher insulin secretion was found for islets coincubated with AMSCs, which was associated with higher stimulation indexes in comparison to other groups $(31,340 \pm 7,769 \mathrm{mU} /$ $\mathrm{ml}$ vs. $50,709 \pm 25,883 \mathrm{mU} / \mathrm{ml}$ at $\mathrm{G} 5$ and $\mathrm{G} 15+\mathrm{Fsk}$, respectively, for islets +AMSCs; $15,614 \pm 14,035 \mathrm{mU} / \mathrm{ml}$ vs. $6,092 \pm 8,112 \mathrm{mU} / \mathrm{ml}$ at G5 and G15 + Fsk, respectively, for islets+BM-MSCs and 23,942 $\pm 6,574 \mathrm{mU} / \mathrm{ml}$ vs. $25,578 \pm 6,402 \mathrm{mU} / \mathrm{ml}$ at G5 and G15+Fsk, respectively, for islets alone). Moreover, islet insulin content was also higher for islets + AMSCs in comparison to other groups $(170,581 \pm 7,769 \mathrm{mU} / \mathrm{ml}$ vs. $191,355 \pm 64,921 \mathrm{mU} / \mathrm{ml}$ at G5 and G15+Fsk, respectively, for islets+AMSCs; $350,440 \pm 67,090 \mathrm{mU} / \mathrm{ml}$ vs. $230,585 \pm 110,273 \mathrm{mU} / \mathrm{ml}$ at G5 and G15+Fsk, respectively, for islets + BM-MSCs and $220,116 \pm 98,565 \mathrm{mU} / \mathrm{ml}$ vs. $29,973 \pm 25,500 \mathrm{mU} / \mathrm{ml}$ at G5 and G15+Fsk, respectively, for islets alone). The insulin content in the islets + AMSCs group was stable and correlated with better cellular survival, but insulin content decreased after glucose stimulation in islets alone and islets + BM-MSC groups (Fig. 2E, F).

\section{In Vivo Assessments}

In Vivo Graft Oxygenation in Diabetic Rats (Fig. 3). A significantly higher $\mathrm{pO}_{2}$ was found in MCDs containing islets coencapsulated with AMSCs in comparison to islets alone at 3 and 4 weeks after transplantation $(p<0.001)$ (Fig. $3 \mathrm{~A})$. The cotransplantation of islets with AMSCs induced a significant increase of the AUC, indicating a better oxygenation, in comparison to islets+BM-MSCs and islets alone (mean AUC of $10.5 \pm 0.8 \% \mathrm{O}_{2} /$ week in comparison to $8.9 \pm 0.5$ and $4.3 \pm 1.1 \% \mathrm{O}_{2}$ /week, respectively, $p<0.01$ ).

\section{In Vivo Assessment in Diabetic Primates}

Metabolic Course After Transplantation. Seven primates out of 10 underwent anticipated implant removal from $24 \mathrm{~h}$ to 29 weeks posttransplantation independent of graft dysfunction. Primate 08-11 died at 17 weeks posttransplantation from small bowel intussusception, primate 09-011 died 1 day after transplantation from severe hypoglycemia, primate 09-182 removed one graft at 19 weeks postimplantation and the other one (last one) at 28 weeks, primate 09-178 removed one graft 1 week posttransplantation, primate 09-183 lost significant body weight (30\%) after STZ treatment, primate 10-037 died from dehydration

\section{FACING PAGE}

Figure 3. In vivo oxygenation of implants containing islets + MSCs and metabolic follow-up of primates. (A) In vivo oxygenation of islets coencapsulated with BM-MSCs or AMSCs versus islets encapsulated alone grafted in diabetic rats. Significantly higher oxygen levels were found in MCDs containing islets and AMSCs in comparison to islets alone at 3 and 4 weeks after implantation $(* p<0.01)$. Gray rectangle represents oxygen levels found in native pancreatic islets. (B) Glycemia courses of primates transplanted with pig islets coencapsulated with BM-MSCs. Reduction of FBG was observed up to 30 weeks postimplantation with a mean FBG of $108 \pm 70 \mathrm{mg} /$ dl. An increase in FBG was observed after graft explantation. (C) Pig islet coencapsulation in MCD with BM-MSCs at 17 weeks postimplantation. (D) Insulin staining on retrieved MCDs containing islets and BM-MSCs at 17 weeks postimplantation. (E) FBG curves of primates transplanted with pig islets coencapsulated with AMSCs. Reduction of FBG was observed up to 32 weeks postimplantation, with mean FBG of $113 \pm 72 \mathrm{mg} / \mathrm{dl}$. Increase in FBG was observed after graft explantation. (F) MCDs containing islets and AMSCs at 29 weeks postimplantation. Note the significant angiogenesis in the periphery of the graft and the absence of fibrosis. (G) Insulin staining on MCDs containing islets and AMSCs retrieved at 29 weeks postimplantation. Note the presence of clusters of insulin-positive cells. The body weight was maintained at $104 \pm 12 \%$ and $114 \pm 19 \%$ of initial body weight at 12 weeks posttransplantation of islets + BM-MSCs or AMSCs, respectively. At 28 weeks posttransplantation, primates grafted with islets + BM-MSCs and islets + AMSCs demonstrated $100 \pm 4 \%$ and $100 \pm 18 \%$ of initial body weight, respectively. Dot lines represent minimal FBG baseline for diabetic primates. 


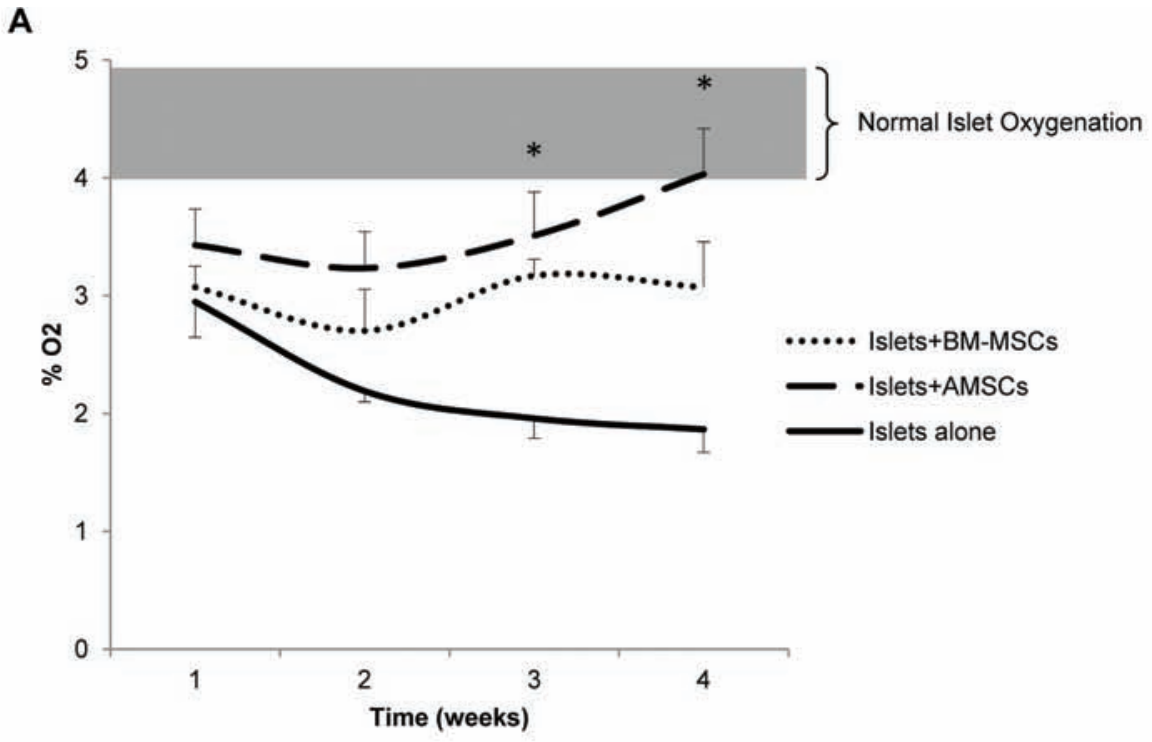

B


E
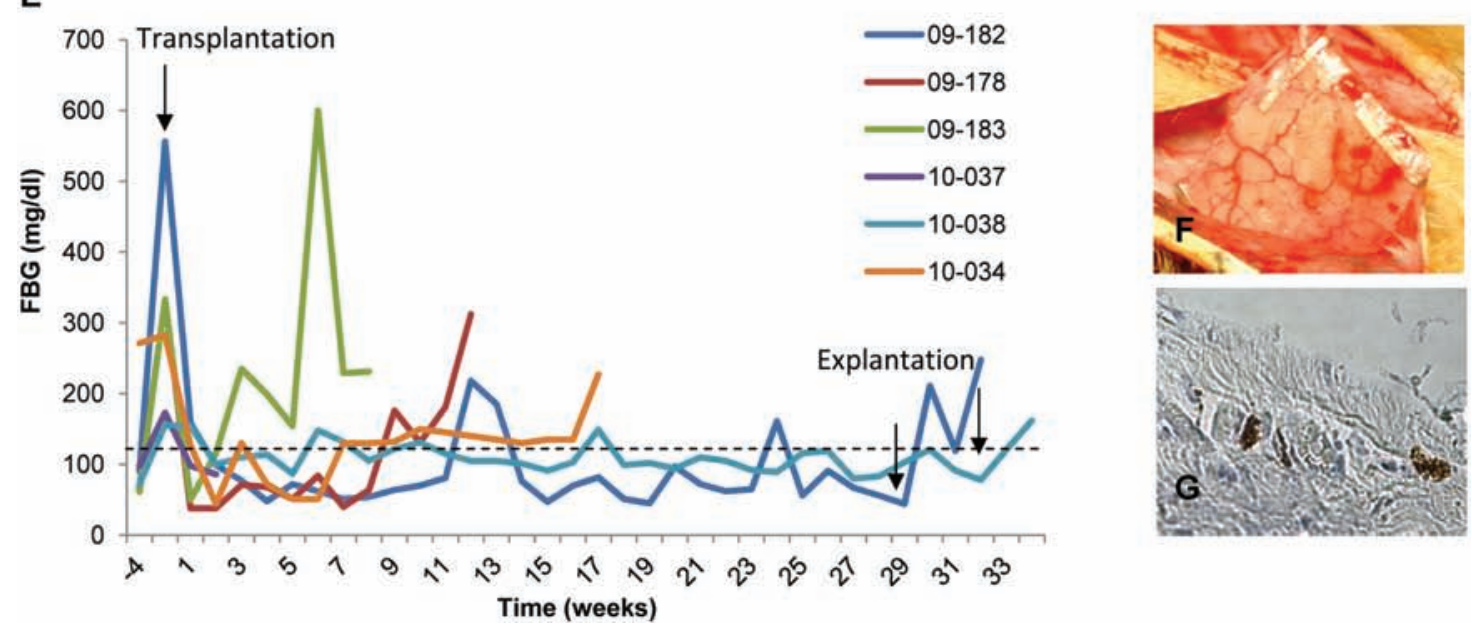


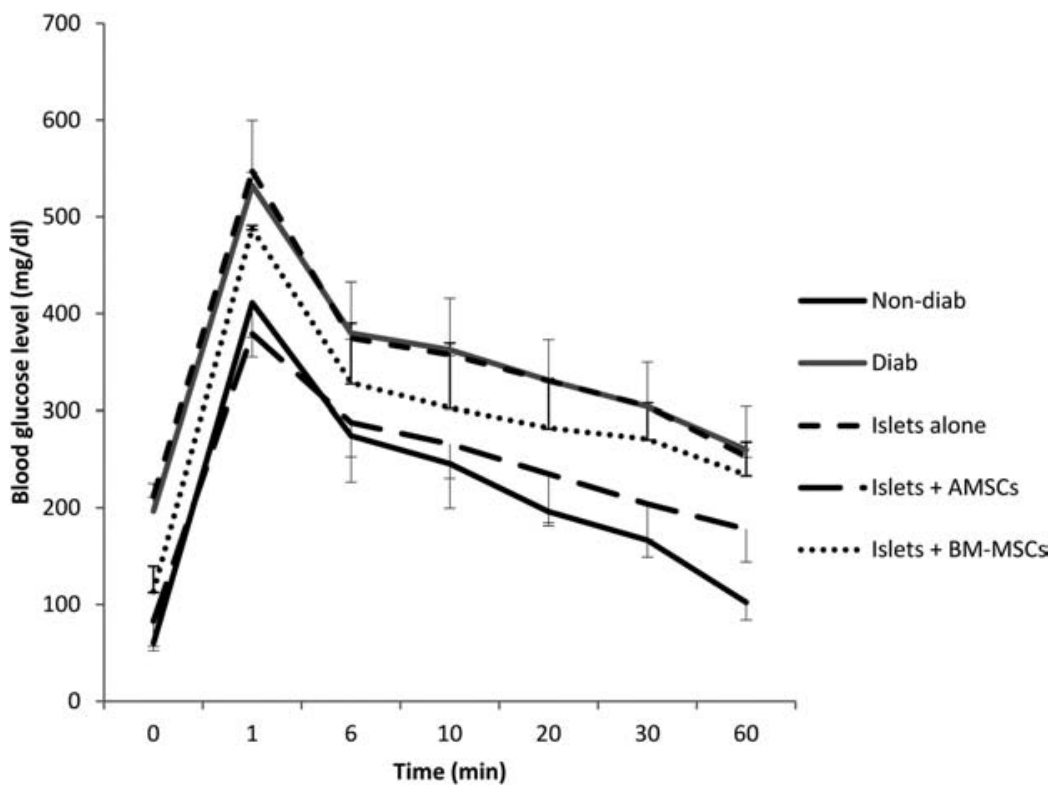

Figure 4. Representative IVGTT curves obtained prior to diabetes induction, before transplantation and at a mean of 28 weeks postimplantation (islets + MSCs) or 22 weeks postimplantation (islets alone). IVGTT, intravenous glucose tolerance test; Non-diab, prior to diabetes induction; Diab, before transplantation and other groups are 22 or 28 weeks postimplantation.

2 weeks after transplantation, and primate 10-034 suffered from hepatic failure following STZ treatment.

Primates transplanted with pig islets coencapsulated with BM-MSCs demonstrated normalized glycemia for up to 31 weeks postimplantation (Fig. 3B, C). Mean FBG and $\mathrm{HbA} 1 \mathrm{c}$ of $93 \mathrm{mg} / \mathrm{dl}$ and $9.2 \%$, respectively, were found at 24 weeks posttransplantation $(n=2$ of 4$)$. The cotransplantation of islets and AMSCs showed a diabetes correction for a maximum of 32 weeks (Fig. 3E, F). At 24 weeks posttransplantation, a mean FBG of $125 \mathrm{mg} / \mathrm{dl}$ and mean $\mathrm{HbA} 1 \mathrm{c}$ of $9.3 \%$ were observed $(n=2$ of 6$)$. Primates previously grafted with islets alone showed mean FBG and $\mathrm{HbA} 1 \mathrm{c}$ of $215 \mathrm{mg} / \mathrm{dl}$ and $9.8 \%$, respectively, at 24 weeks posttransplantation ( $n=5$ of 5) (9).

At 28 weeks postimplantation, a mean $\mathrm{HbA1c}$ of $7.4 \%$ and $8.1 \%$ was observed for islets + BM-MSCs and islets+AMSCs, respectively. These HbA1c were lower than those observed in primates previously grafted with islets alone (mean of 10.9\%) (9).

Representative IVGTT curves prior to STZ treatment, prior to transplantation, and at a mean of 28 weeks posttransplantation (islets $+\mathrm{MSCs}$ ) are shown in Figure 4. A maximal insulin secretion in primate serum of 44.6 and $27.4 \mu \mathrm{U} / \mathrm{ml}$ was measured for islets+BM-MSCs and islets + AMSCs, respectively, at 20 min post-glucose injection, in comparison to $1.7 \mu \mathrm{U} / \mathrm{ml}$ for islets alone (data not shown).

After graft removal, glycemia increased to pathologic levels in all transplanted primates $(208 \pm 109 \mathrm{mg} / \mathrm{dl})$.

The body weight was maintained at $104 \pm 12 \%$ and $114 \pm 19 \%$ of initial body weight at 12 weeks posttransplantation of islets+BM-MSCs or AMSCs, respectively. At 28 weeks posttransplantation, primates grafted with islets + BM-MSCs and islets + AMSCs demonstrated 100 \pm $4 \%$ and $100 \pm 18 \%$ of initial body weight, respectively.

After graft dysfunction or removal by the primate, insulin staining was performed on explanted grafts. Insulin-positive cells were observed up to 30 weeks postimplantation for islets + BM-MSCs (Fig. 3D) and up to 29 weeks postimplantation for islets + AMSCs (Fig. 3G). In contrast, only small clusters of $\beta$-cells were found in the pancreas of primates, confirming the destruction of $\beta$-cells by STZ treatment and the diabetic state.

Humoral Response After Transplantation. The humoral response against pig islets was assessed by analysis of sera from transplanted primates by flow cytometry. The

\section{FACING PAGE}

Figure 5. Immunologic courses of primates. (A) Anti-pig antibodies. All tested primates showed increased levels of anti-pig antibodies (both IgM and IgG). No significant difference was found between tested groups. Results are presented as the mean of IgM and IgG levels per tested group. (B) Primate antibodies directed specifically against $\alpha$-galactosyl ( $\alpha$-Gal) epitope. No significant difference was found between tested groups. Results are presented as the mean of the levels of anti-pig antibodies directed against $\alpha$-Gal epitope per tested group. (C) Cytotoxicity of anti-pig antibodies for transplanted primates at 30 and 120 days posttransplantation. No significant difference was found between tested groups. Results are presented as the mean of cytotoxicity levels per tested group. 

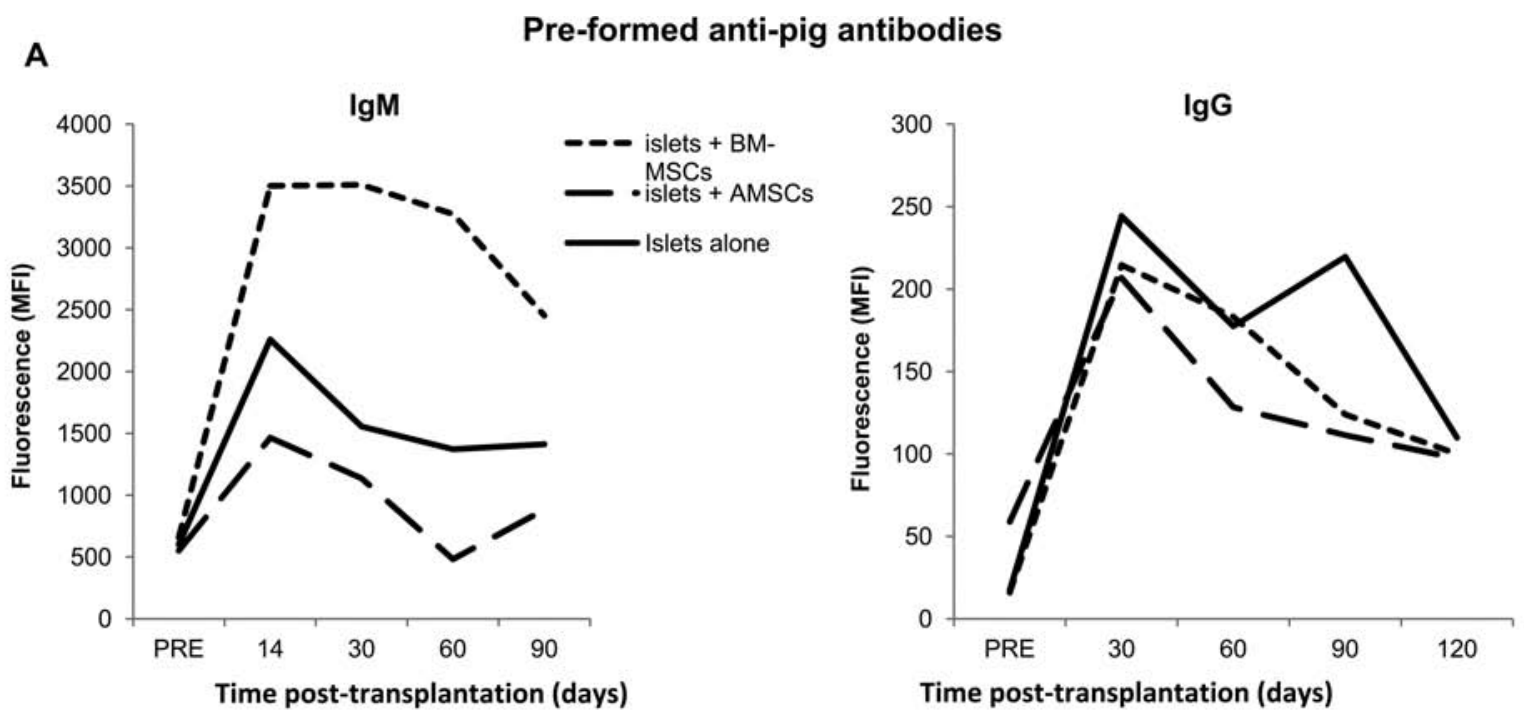

\section{B}

Gal specificity

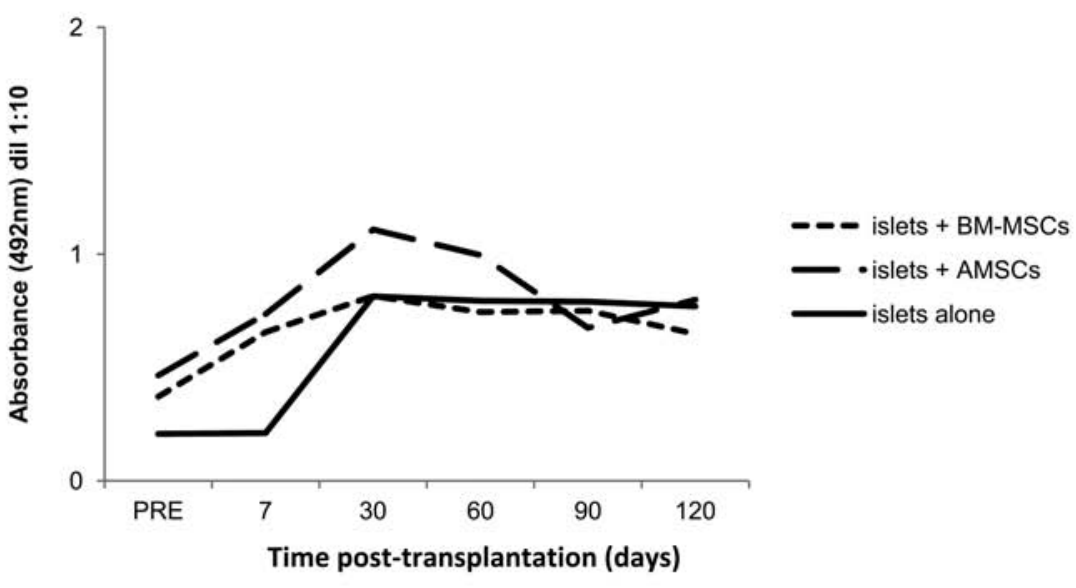

C

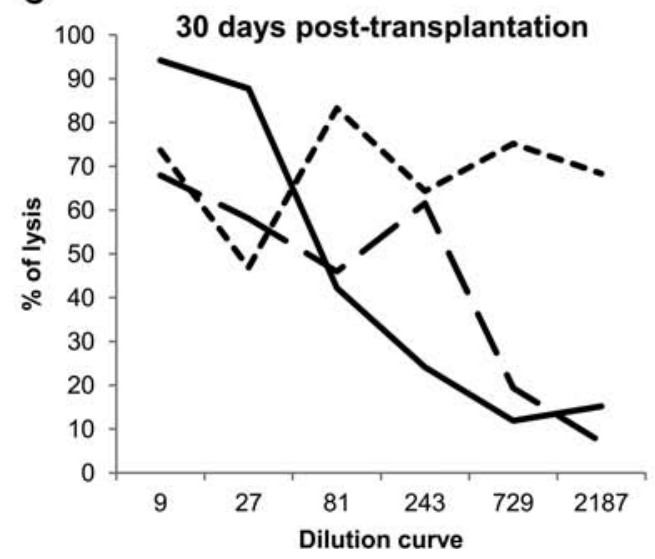

\section{Cytotoxicity}

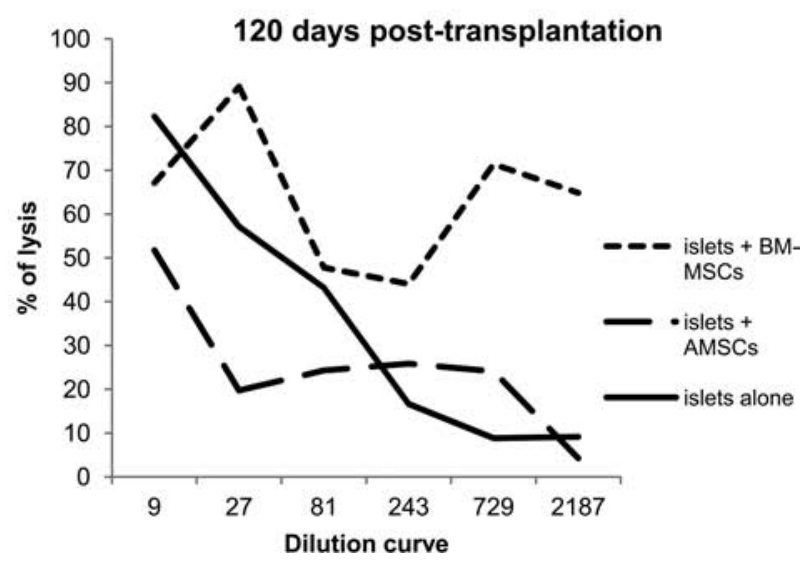


presence of preformed anti-pig antibodies was confirmed by the detection of both IgM and IgG in primates' sera prior to transplantation. The levels of anti-pig antibodies increased after transplantation in all primates. $\operatorname{IgM}$ and IgG reached a maximum at day 14 and day 30, respectively, and decreased thereafter (Fig. 5A). Some of the antibodies detected were directed against the $\alpha$-Gal epitope, as confirmed by ELISA (Fig. 5B). The ability of immunoglobulins to activate complement was confirmed by fluorescence-activated cell sorting (FACS) at days 30 and 120 posttransplantation (Fig. 5C). A reduction of the cytotoxicity titer of anti-pig antibodies was found at 120 days. Sera from primates previously grafted with islets alone (9) were also tested as controls. No difference in terms of anti-pig antibodies and cytotoxicity was observed between experimental groups (Fig. 5B, C).

Assessment of Implant Neovascularization. The neovascularization of implants was compared for each group by histomorphometry analysis on the thin membrane surrounding MCDs (Fig. 6).

In rats, similar numbers of vessels per area and of VEGF-expressing cells per area were found in the periphery of islets coencapsulated with BM-MSCs or AMSCs at 4 weeks posttransplantation. In contrast, a significantly higher number of vessels per area as well as a higher number of VEGF-expressing cells per area were found in periphery of islets coencapsulated with either AMSCs or BM-MSCs in comparison to islets alone $(p<0.05)$ (data not shown).

In primates, a significantly higher number of vessels per area (Fig. 6A) as well as a higher number of vWF-positive cells per area (Fig. 6E) were found in the periphery of islets coencapsulated with AMSCs (mean of 18 weeks postimplantation) (Fig. 6D, H, respectively) or BM-MSCs (mean of 24 weeks postimplantation) (Fig. 6C, G, respectively) in comparison to islets alone (mean of 28 weeks postimplantation) ( $p<0.01$ ) (Fig. 6B, F, respectively). In contrast, no significant difference was observed between the AMSCs + islets and BM-MSCs + islets groups.
A significantly higher number of VEGF-positive cells per area (Fig. 6I) was quantified in the periphery of islets coencapsulated with AMSCs (Fig. 6L) in comparison to islets alone (Fig. 6J) and islets coencapsulated with BM-MSCs $(p<0.001)$ (Fig. 6K).

However, no significant difference in the vascular density around explanted grafts was found between tested groups (Fig. 6M).

\section{DISCUSSION}

Despite continuous improvements in the field of encapsulated islet transplantation, bioartificial pancreases still need to be improved to support graft neovascularization and oxygenation, reduce posttransplantation stress, support islet function, and ensure long-term graft survival and function.

Since oxygen levels inside a subcutaneous bioartificial pancreas are not sufficient to ensure optimal survival and function of encapsulated islets $\left(\sim 2 \% \mathrm{O}_{2}\right.$ vs. $\sim 5 \% \mathrm{O}_{2}$ for encapsulated islets vs. native pancreatic islets, respectively) $(3,4,29)$, the use of MSCs was proposed to improve implant vascularization and, consequently, graft oxygenation.

The first question addressed in this work was to investigate the impact of MSC supplementation on islet survival and function. We showed that AMSCs improved in vitro islet survival both in normo- and hyperglycemic conditions. This was associated with better islet function. After glucose stimulation, islets showed increased insulin secretion, but stable insulin content (in normo- and hyperglycemic conditions) was observed only for islets coincubated with AMSCs. Insulin content in islets alone or islets+BM-MSCs decreased $30 \%$ to $90 \%$ after glucose stimulation. These results are in accordance with other studies published recently. BM-MSCs demonstrated in vitro improvement of islet viability and insulin secretion, associated with increased levels of antiapoptotic molecules (16).

However, the cellular encapsulation can modify the metabolism of both MSCs and islets macroencapsulated in the MCD.

\section{FACING PAGE}

Figure 6. Quantification of the number of vessels and vascular density and VEGF- and vWF-expressing cells in the periphery of MCDs implanted in diabetic primates: islets alone versus BM-MSCs and AMSCs. (A) A significantly higher number of vessels per area was found in the periphery of islets coencapsulated with BM-MSCs and AMSCs in comparison to islets alone (*p<0.01). No significant difference was found between islets + BM-MSCs and islets + AMSCs. (B) Masson's trichrome staining on MCD containing islets alone at 22 weeks postimplantation. (C) Masson's trichrome staining on MCDs containing islets + BM-MSCs at 17 weeks postimplantation. (D) Masson's trichrome staining on MCDs containing islets + AMSCs at 18 weeks postimplantation. (E) A significantly higher number of vWF-expressing cells per area was found in the periphery of islets coencapsulated with BM-MSCs and AMSCs in comparison to islets alone $\left({ }^{*} p<0.01\right)$. No significant difference was found between islets $+\mathrm{BM}-\mathrm{MSCs}$ and islets $+\mathrm{AMSCs}$. (F) vWF staining on MCDs containing islets alone at 22 weeks postimplantation. $(\mathrm{G}) \mathrm{vWF}$ staining on MCDs containing islets + BM-MSCs at 17 weeks postimplantation. (H) vWF staining on MCDs containing islets + AMSCs at 18 weeks postimplantation. (I) The cotransplantation of islets and AMSCs demonstrated a significantly higher number of VEGF-positive cells per area in comparison to islets alone and islets + BM-MSCs $(* * p<0.001)$. (J) VEGF staining on MCDs containing islets alone at 22 weeks postimplantation, (K) islets + BM-MSCs at 17 weeks postimplantation, and (L) islets + AMSCs at 18 weeks postimplantation. (M) No significant difference in the vascular density was found between tested groups. Results are presented as means \pm SD. 
A
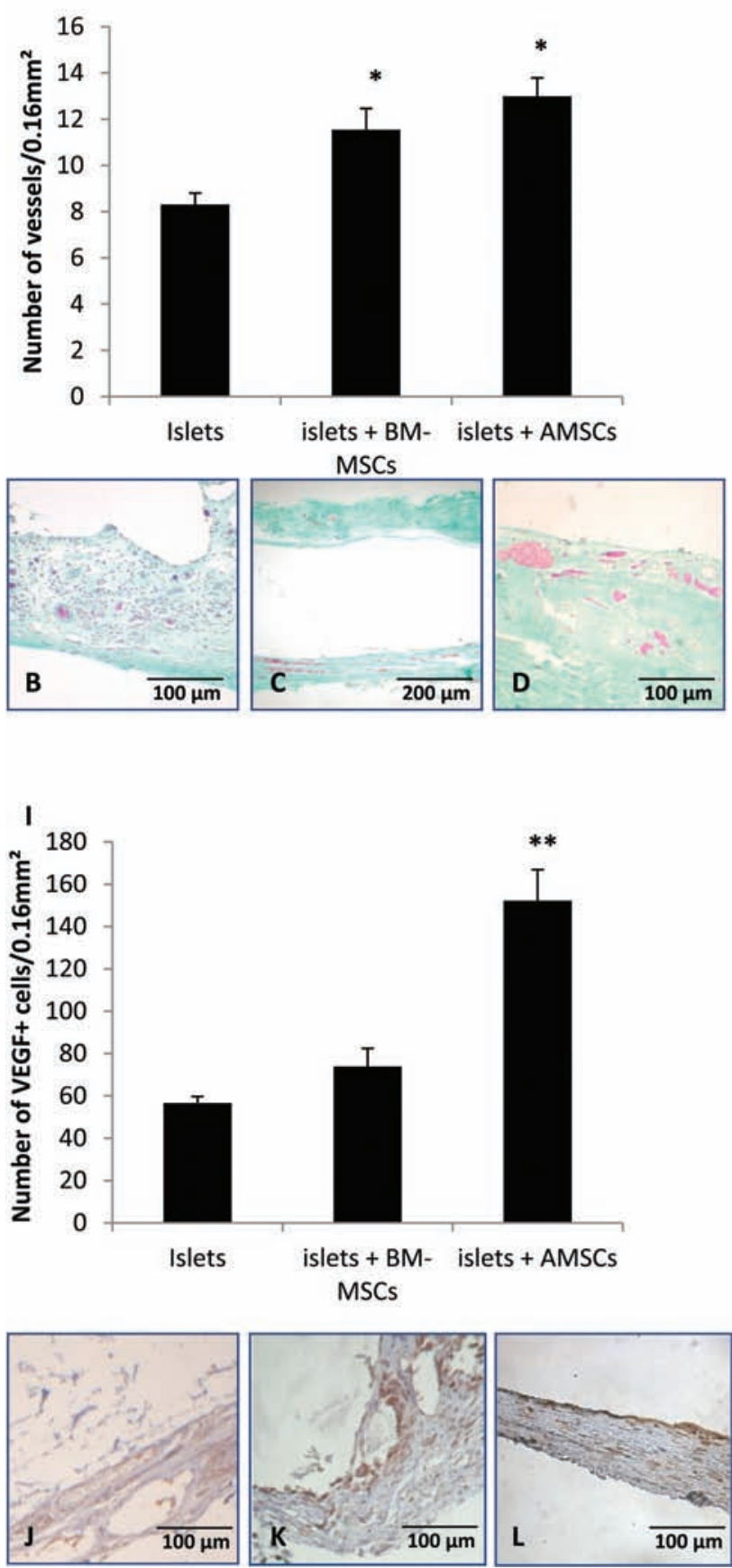

By increasing the number of cells per volume unit, the coencapsulation of MSCs and islets could increase the oxygen consumption rate by encapsulated cells and therefore limit the amount of oxygen available for transplanted islets. We confirmed in vitro that islets coincubated with AMSCs showed an increased oxygen consumption rate in comparison to islets alone, both in normo- and hyperglycemic conditions. In contrast, increased oxygen consumption by islets + BM-MSCs versus islets alone was
E
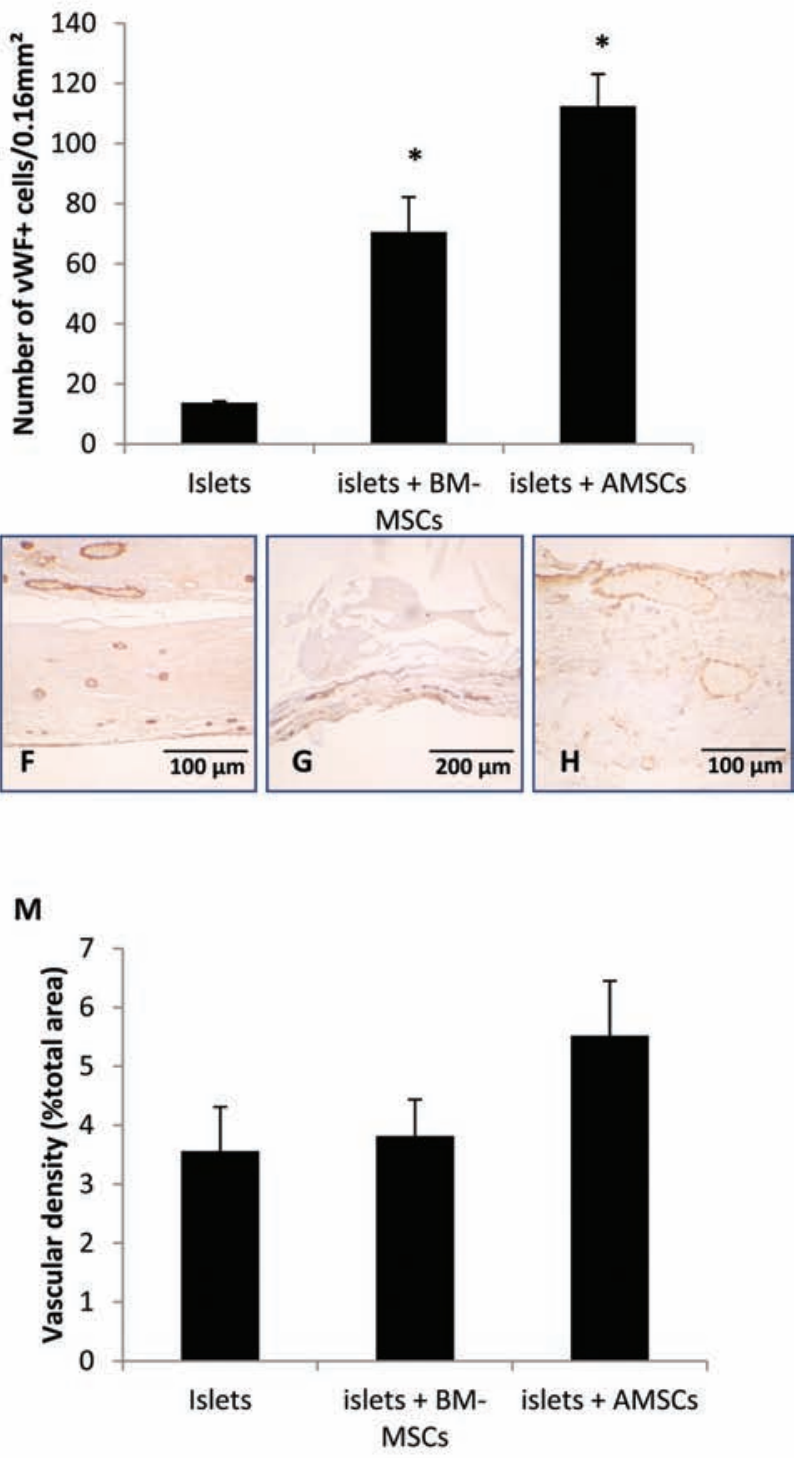

observed only in hyperglycemic conditions. This discrepancy between both cell types can be explained by a higher metabolism of AMSCs than BM-MSCs, especially in hyperglycemic conditions, associated with a higher cellular turnover. Adipose tissue-derived stem cells showed a higher proliferation capacity in comparison to BM-MSCs $(6.1 \pm 2.3$ days vs. $9.0 \pm 1.9$ days between each passage for AMSCs vs. BM-MSCs, respectively) (25). Although encapsulated AMSCs alone demonstrated 
a beneficial effect on implant neovascularization and oxygenation in vivo (29), their significant oxygen consumption could limit the amount of oxygen available for coencapsulated islets.

Consequently, it was important to study the impact of islet and MSC coencapsulation and transplantation on graft vascularization and oxygenation. In contrast to an increase in oxygen consumption by MSCs in vitro, improved vascularization of the MCD (number of vessels and number of VEGF-expressing cells per area) was found in the short term (4 weeks) in vivo for islets cotransplanted with either BM-MSCs or AMSCs in comparison to islets alone. This higher vascularization was correlated with higher graft oxygen levels (3\% and 3.6\% $\mathrm{O}_{2}$ for islets+BM-MSCs or AMSCs, respectively, vs. $2.2 \%$ for islets alone). Improved graft vascularization by MSCs was also found in a model of cotransplantation of islets and kidney-derived MSCs or AMSCs in diabetic animals in comparison to islets alone $(18,21)$. Improved early graft vascularization and oxygenation are particularly important because they can prevent cell loss during the first days postimplantation. Thirty percent of transplanted islets can be lost during the first week posttransplantation (before the establishment of a vascular network surrounding the graft) due to cellular stress following hypoxia (11).

The discrepancy between in vitro (a higher oxygen consumption by AMSCs) and in vivo (improvement of MCD oxygenation with AMSCs) results can be explained by a lower cellular turnover of encapsulated AMSCs after subcutaneous transplantation, which can induce a reduction of oxygen consumption by AMSCs and sensitivity of the host subcutaneous tissue to the VEGF (released by encapsulated AMSCs) with regard to improved neovascularization and consequently graft oxygenation. Although the early oxygenation of the graft can be improved by AMSCs in the MCD, the beneficial effects on graft function and diabetes correction remains unclear with longterm suboptimal vascularization.

To assess the impact of MSCs on islet survival and function as well as on graft vascularization in vivo long term, pig islets were coencapsulated with BM-MSCs or AMSCs and implanted subcutaneously in STZ-induced diabetic primates. The results were compared to primates previously grafted with islets encapsulated alone (9). Greater long-term vascularization was found in the periphery of islets coencapsulated with MSCs in comparison to islets alone (mean explantation time of 24 weeks). Although islets cotransplanted with MSCs showed a higher number of vessels surrounding the graft, no significant increase of the vascular density was found in comparison with islets alone. This can be explained by a higher concentration of small vessels surrounding implants made of islets + MSCs in comparison to MCDs containing islets alone (low number of large vessels). An increased number of vessels, even smaller, can probably facilitate molecule diffusion through the alginate membrane. Seven of the 10 transplanted primates underwent anticipated implant removal for reasons independent of graft function or biocompatibility. The analysis of the four other primates showed that the cotransplantation of islets with MSCs improved slightly graft survival and function. A mean of 23 and 30 weeks of diabetes correction was found for primates transplanted with islets and AMSCs or BM-MSCs, respectively, in contrast to 23 weeks for primates transplanted with islets alone, associated with a maximum graft function at 32 and 31 weeks for islets cotransplanted with AMSCs and BM-MSCs, respectively, in contrast to 28 weeks for islets transplanted alone. In addition, better diabetes correction was found for primates transplanted with islets coencapsulated with MSCs in comparison to islets encapsulated alone as revealed by a better correction of $\mathrm{HbA1c}$. Although the diabetes correction was prolonged by the cotransplantation of MSCs in the MCD, this higher vascularization did not significantly sustain the diabetes correction long term (up to 8 months).

One explanation of this limited diabetes correction could be a higher immunization by cotransplanted MSCs in the MCD. Although the alginate membrane of the MCD is impermeable to molecules of $150 \mathrm{kDa}$ (molecular weight of an $\mathrm{IgG}$ ), peptides of low molecular weight (36 and $75 \mathrm{kDa}$ ) were shown to cross the alginate barrier $(9,30)$. After the transplantation of encapsulated pig islets in diabetic primates, the production of anti-pig antibodies has been observed (9). These antibodies were mainly directed against the highly immunogenic $\alpha$-galactosyl antigen (9). Porcine MSCs, like pig islets, were shown to express $\alpha$-galactosyl antigen (19). In this study, no increase of anti-pig antibodies ( $\operatorname{IgM} / \operatorname{IgG}$ ) was found in the sera of primates transplanted with islets + MSCs in comparison to islets alone. These results indicate that confluent encapsulated MSCs do not grow (outside MCD) long term. Moreover, no difference in antibody cytotoxicity was found between tested groups.

In conclusion, improvement of a bioartificial pancreas can be obtained through cotransplantation of MSCs and islets. Coincubation of islets and AMSCs increased islet survival and function in vitro. In addition, the cotransplantation of islets with BM-MSCs and AMSCs increased subcutaneous bioartificial pancreas vascularization and oxygenation in the short term (4 weeks), consequently reducing the cellular stress occurring in the first days posttransplantation. In a preclinical model, cotransplantation of islets and MSCs improved graft vascularization without increased production of anti-pig antibodies, showing that the procedure did not induce hyperimmunization of the recipient. However, it only slightly 
improved graft function and survival long term. The problem that remains to be overcome in islet transplantation is the identification of a renewable and long-acting $\beta$-cell source. MSCs could be expanded and differentiated, thus potentially constituting a continuous source of insulin-producing cells. Further investigations still need to be done in this field.

ACKNOWLEDGMENTS: We are grateful to D. Xhema, P.Y. Adnet, Myriam Nenquin, and Gabriel Padron for technical assistance and P. Segers and E. Legrand for editorial and logistic help, respectively. We thank FMC BioPolymer (Drammen, Norway) for providing alginate. This work was supported by a European grant titled Xenome (UE LSHB-CT-2006-037377) and by the national fund "Fonds national de la recherche scientifique" (FRS-FNRS; FRSM 3.4517.11). Sophie Vériter is an aspirant (doctoral candidate) of FRS-FNRS. The authors declare no conflicts of interest.

\section{REFERENCES}

1. Alejandro, R.; Barton, F. B.; Hering, B. J.; Wease, S. 2008 Update from the Collaborative Islet Transplant Registry. Transplantation 86:1783-1788; 2008.

2. Baudelet, C.; Gallez, B. Effect of anesthesia on the signal intensity in tumors using BOLD-MRI: Comparison with flow measurements by Laser Doppler flowmetry and oxygen measurements by luminescence-based probes. Magn. Reson. Imaging 22:905-912; 2004.

3. Carlsson, P. O.; Liss, P.; Andersson, A.; Jansson, L. Measurements of oxygen tension in native and transplanted rat pancreatic islets. Diabetes 47:1027-1032; 1998.

4. Carlsson, P. O.; Palm, F. Oxygen tension in isolated transplanted rat islets and in islets of rat whole-pancreas transplants. Transpl. Int. 15:581-585; 2002.

5. Diepart, C.; Verrax, J.; Calderon, P. B.; Feron, O.; Jordan, B. F.; Gallez, B. Comparison of methods for measuring oxygen consumption in tumor cells in vitro. Anal. Biochem. 396:250-256; 2010.

6. Dominici, M.; Le Blanc, K.; Mueller, I.; Slaper-Cortenbach, I.; Marini, F.; Krause, D.; Deans, R.; Keating, A.; Prockop, D.; Horwitz, E. Minimal criteria for defining multipotent mesenchymal stromal cells. The International Society for Cellular Therapy position statement. Cytotherapy 8:315$317 ; 2006$.

7. Dufrane, D.; Cornu, O.; Delloye, C.; Schneider, Y. J. Physical and chemical processing for a human dura mater substitute. Biomaterials 23:2979-2988; 2002.

8. Dufrane, D.; D’hoore, W.; Goebbels, R. M.; Saliez, A.; Guiot, Y.; Gianello, P. Parameters favouring successful adult pig islet isolations for xenotransplantation in pig-toprimate models. Xenotransplantation 13:204-214; 2006.

9. Dufrane, D.; Goebbels, R. M.; Gianello, P. Alginate macroencapsulation of pig islets allows correction of streptozotocin-induced diabetes in primates up to 6 months without immunosuppression. Transplantation 90:1054-1062; 2010.

10. Dufrane, D.; Goebbels, R. M.; Saliez, A.; Guiot, Y.; Gianello, P. Six-month survival of microencapsulated pig islets and alginate biocompatibility in primates: Proof of concept. Transplantation 81:1345-1353; 2006.

11. Dufrane, D.; van Steenberghe, M.; Goebbels, R. M.; Saliez, A.; Guiot, Y.; Gianello, P. The influence of implantation site on the biocompatibility and survival of alginate encapsulated pig islets in rats. Biomaterials 27:3201-3208; 2006.

12. Dufrane, D.; van Steenberghe, M.; Guiot, Y.; Goebbels, R. M.; Saliez, A.; Gianello, P. Streptozotocin-induced diabetes in large animals (pigs/primates): Role of GLUT2 transporter and beta-cell plasticity. Transplantation $81: 36-45 ; 2006$.

13. Gallez, B.; Jordan, B. F.; Baudelet, C.; Misson, P. D. Pharmacological modifications of the partial pressure of oxygen in murine tumors: Evaluation using in vivo EPR oximetry. Magn. Reson. Med. 42:627-630; 1999.

14. Hirshberg, B.; Rother, K. I.; Digon, B. J., III; Lee, J.; Gaglia, J. L.; Hines, K.; Read, E. J.; Chang, R.; Wood, B. J.; Harlan, D. M. Benefits and risks of solitary islet transplantation for type 1 diabetes using steroid-sparing immunosuppression: The National Institutes of Health experience. Diabetes Care 26:3288-3295; 2003.

15. Jordan, B. F.; Peeterbroeck, J.; Karroum, O.; Diepart, C.; Magat, J.; Gregoire, V.; Gallez, B. Captopril and S-nitrosocaptopril as potent radiosensitizers: Comparative study and underlying mechanisms. Cancer Lett. 293:213$219 ; 2010$.

16. Karaoz, E.; Genc, Z. S.; Demircan, P. C.; Aksoy, A.; Duruksu, G. Protection of rat pancreatic islet function and viability by coculture with rat bone marrow-derived mesenchymal stem cells. Cell Death. Dis. 1:e36; 2010.

17. Mineo, D.; Ricordi, C.; Xu, X.; Pileggi, A.; Garcia-Morales, R.; Khan, A.; Baidal, D. A.; Han, D.; Monroy, K.; Miller, J.; Pugliese, A.; Froud, T.; Inverardi, L.; Kenyon, N. S.; Alejandro, R. Combined islet and hematopoietic stem cell allotransplantation: A clinical pilot trial to induce chimerism and graft tolerance. Am. J. Transplant. 8:1262-1274; 2008.

18. Ohmura, Y.; Tanemura, M.; Kawaguchi, N.; Machida, T.; Tanida, T.; Deguchi, T.; Wada, H.; Kobayashi, S.; Marubashi, S.; Eguchi, H.; Takeda, Y.; Matsuura, N.; Ito, T.; Nagano, H.; Doki, Y.; Mori, M. Combined transplantation of pancreatic islets and adipose tissue-derived stem cells enhances the survival and insulin function of islet grafts in diabetic mice. Transplantation 90:1366-1373; 2010.

19. Poncelet, A. J.; Denis, D.; Gianello, P. Cellular xenotransplantation. Curr. Opin. Organ Transplant. 14:168-174; 2009.

20. Poncelet, A. J.; Vercruysse, J.; Saliez, A.; Gianello, P. Although pig allogeneic mesenchymal stem cells are not immunogenic in vitro, intracardiac injection elicits an immune response in vivo. Transplantation 83:783-790; 2007.

21. Rackham, C. L.; Chagastelles, P. C.; Nardi, N. B.; HaugeEvans, A. C.; Jones, P. M.; King, A. J. Co-transplantation of mesenchymal stem cells maintains islet organisation and morphology in mice. Diabetologia 54:1127-1135; 2011.

22. Ryan, E. A.; Lakey, J. R.; Paty, B. W.; Imes, S.; Korbutt, G. S.; Kneteman, N. M.; Bigam, D.; Rajotte, R. V.; Shapiro, A. M. Successful islet transplantation: Continued insulin reserve provides long-term glycemic control. Diabetes 51:2148-2157; 2002.

23. Ryan, E. A.; Paty, B. W.; Senior, P. A.; Bigam, D.; Alfadhli, E.; Kneteman, N. M.; Lakey, J. R.; Shapiro, A. M. Fiveyear follow-up after clinical islet transplantation. Diabetes 54:2060-2069; 2005.

24. Schecroun, N.; Delloye, C. In vitro growth and osteoblastic differentiation of human bone marrow stromal cells supported by autologous plasma. Bone 35:517-524; 2004. 
25. Schubert, T.; Xhema, D.; Veriter, S.; Schubert, M.; Behets, C.; Delloye, C.; Gianello, P.; Dufrane, D. The enhanced performance of bone allografts using osteogenic-differentiated adipose-derived mesenchymal stem cells. Biomaterials 32: 8880-8891; 2011.

26. Schuleri, K. H.; Feigenbaum, G. S.; Centola, M.; Weiss, E. S.; Zimmet, J. M.; Turney, J.; Kellner, J.; Zviman, M. M.; Hatzistergos, K. E.; Detrick, B.; Conte, J. V.; McNiece, I.; Steenbergen, C.; Lardo, A. C.; Hare, J. M. Autologous mesenchymal stem cells produce reverse remodelling in chronic ischaemic cardiomyopathy. Eur. Heart. J. 30:27222732; 2009 .

27. Shapiro, A. M.; Lakey, J. R.; Ryan, E. A.; Korbutt, G. S.; Toth, E.; Warnock, G. L.; Kneteman, N. M.; Rajotte, R. V. Islet transplantation in seven patients with type 1 diabetes mellitus using a glucocorticoid-free immunosuppressive regimen. N. Engl. J. Med. 343:230-238; 2000.

28. Shapiro, A. M.; Ricordi, C.; Hering, B. J.; Auchincloss, H.; Lindblad, R.; Robertson, R. P.; Secchi, A.; Brendel, M. D.;
Berney, T.; Brennan, D. C.; Cagliero, E.; Alejandro, R.; Ryan, E. A.; DiMercurio, B.; Morel, P.; Polonsky, K. S.; Reems, J. A.; Bretzel, R. G.; Bertuzzi, F.; Froud, T.; Kandaswamy, R.; Sutherland, D. E.; Eisenbarth, G.; Segal, M.; Preiksaitis, J.; Korbutt, G. S.; Barton, F. B.; Viviano, L.; Seyfert-Margolis, V.; Bluestone, J.; Lakey, J. R. International trial of the Edmonton protocol for islet transplantation. N. Engl. J. Med. 355:1318-1330; 2006.

29. Veriter, S.; Aouassar, N.; Adnet, P. Y.; Paridaens, M. S.; Stuckman, C.; Jordan, B.; Karroum, O.; Gallez, B.; Gianello, P.; Dufrane, D. The impact of hyperglycemia and the presence of encapsulated islets on oxygenation within a bioartificial pancreas in the presence of mesenchymal stem cells in a diabetic Wistar rat model. Biomaterials 32:5945-5956; 2011.

30. Veriter, S.; Mergen, J.; Goebbels, R. M.; Aouassar, N.; Gregoire, C.; Jordan, B.; Leveque, P.; Gallez, B.; Gianello, P.; Dufrane, D. In vivo selection of biocompatible alginates for islet encapsulation and subcutaneous transplantation. Tissue Eng. Part A 16:1503-1513; 2010. 